\title{
Distribution of incident lions and retained dose analysis for a wedge- shaped target in plasma source ion implantation
}

\author{
Shamim M. Malik, D. E. Muller, K. Sridharan, R. P. Fetherston, Ngoc Tran, \\ and J. R. Conrad \\ Department of Nuclear Engineering and Engineering Physics, 1500 Johnson Drive, University \\ of Wisconsin, Madison, Wisconsin 53706
}

(Received 10 June 1994; accepted for publication 13 October 1994)

\begin{abstract}
A wedge-shaped target was implanted with nitrogen ions using the plasma source ion implantation process, in order to understand the effects of the target edges on the energy and fluence distribution of incident ions. Experimental measurements and analysis of retained dose on silicon samples affixed on the surface of the target, showed results consistent with those predicted by theoretical models. Higher retained dose and greater implantation depths were observed in the vicinity of the edge contained by the normal angle as compared to the edges contained by the acute angles. The target face with smaller area accumulated, on the average, higher dose compared to the face with the larger area. (c) 1995 American Institute of Physics.
\end{abstract}

\section{INTRODUCTION}

Plasma source ion implantation (PSII), is a non-line-ofsight technique for surface modification of materials. ${ }^{1,2}$ In PSII, the target to be implanted is installed in a chamber which is evacuated to a base pressure of about $10^{-7}$ Torr. A flow of the gas species desired for implantation is established in the chamber to provide a pressure on the order of $10^{-4}$ Torr. The plasma is generated by the ionization of the gas using either the energetic primary electron impact, radiofrequency (RF) or glow discharge methods. A train of negative high voltage pulses (type $1-100 \mathrm{keV}, 1-50 \mu$ s pulse width and frequency $\approx 100 \mathrm{~Hz}$ ) are applied to the target immersed in the plasma. The resulting electric field accelerates the ions in the plasma to high cnergies toward the target surface. These high energy ions penetrate the target surface producing industrially relevant surface properties in a wide range of engineering materials. The PSII process has been shown to be effective in improving the hardness, tribological characteristics, and corrosion resistance of test flats of engineering materials as well as in extending the service life of engineering tools and components. There is growing worldwide interest in PSII process due to its versatility in surface modification of wide range of materials. ${ }^{3}$

The effect of target shape on the structure of the sheath encapsulating the target and the spatial distribution of the ions incident on the surface of the target in PSII has received some attention in previous studies. A model for sheath expansion behavior for planar, cylindrical, and spherical targets was developed by Scheuer et al. ${ }^{4}$ This model used the Child-Langmuir law as the basis for prediction of the sheath propagation. It was shown that the temporal sheath expansion was fastest for the planar target and slowest for the spherical target. Experimental measurements and calculations for spatial and temporal sheath expansion for cylindrical and spherical geometries performed by Shamim et al. ${ }^{5}$ were in agreement with this model. In another investigation on the dynamic behavior of the sheath in planar targets, Shamim et al. ${ }^{6}$ observed that the sheath forms in ellipsoidlike contours which stretch along the plane of the target. At a distance approximately equal to one diameter of a planar disk from its surface, the geometrical effects of the target were observed to be negligible and the sheath edge position was almost stationary. Watterson ${ }^{7}$ numerically simulated the Child-Langmuir sheath structure around square and knifeshaped edges and found that the ion impact rate for the knife edge was higher than for the square edge. The angle of incidence with the plane of symmetry of the wedge was calculated and its direction was found to change from perpendicular to parallel to the surface of target at the sharp edge. Consequently, the ions impinging at non-normal incidence could reduce the implantation depth and increase the sputtering near the edges.

The present investigation of the PSII process inyolving a wedge-shaped target was undertaken as an experimental verification of the above studies and also because of the relevance of this shape to industrial tools and dies. This study has been targeted to shed light on how to use the PSII technology effectively for these targets as well as to provide a better understanding of the sheath physics related to PSII.

\section{EXPERIMENTAL PROCEDURE}

A meter cube vacuum chamber ${ }^{8}$ equipped with arrays of permanent magnets in its walls was used for this investigation. The target to be implanted was an aluminum prismoidal wedge, the rectangular faces of which were covered with mild steel sheets, $3.17 \mathrm{~mm}$ in thickness. The faces of the wedge encompassed $30^{\circ}, 60^{\circ}$, and $90^{\circ}$ angles and the sides were 85,148 , and $172 \mathrm{~mm}$ in length. For electrical contact, the target was connected to the copper feed-through with an aluminum rod. Glass was used to cover the supporting structure. A schematic illustration of the experimental setup is shown in Fig. 1. The chamber was pumped down to the base pressure of $10^{-6}$ Torr. A nitrogen plasma was generated in the chamber using a filament source. The net ion current to the target was measured with a Rogowski transformer.

Initial experiments were performed by affixing a number of well polished mild steel samples along the faces of the wedge. The entire assembly was implanted with nitrogen 


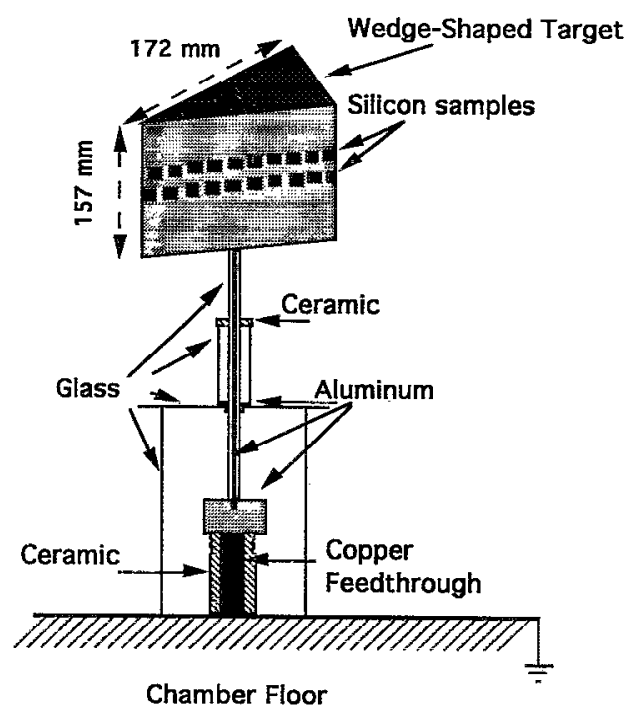

FIG. 1. Experimental arrangement for implanting the wedge-shaped target.

ions at energy of $40 \mathrm{keV}$ to a dose of $10^{18}$ ions $/ \mathrm{cm}^{2}$. Subsequent scanning auger microscopy (SAM) analysis of the near surface of the steel samples did not reveal any significant retention of nitrogen, presumably due to effusion of nitrogen from the surface. A similar observation has been reported by Goode and Baumvol. ${ }^{9}$ Consequently, the present investigation was performed by implanting silicon samples. To ensure good conductivity the samples were affixed to the surface of the steel sheets with silver paint and conductive double-sided carbon tape. A small area on each silicon sample was covered with a titanium dioxide mask in order to measure the depth sputtered during implantation.

The experiments were performed at two independent implantation conditions at retained doses of $1.5 \times 10^{17}$ ions $/ \mathrm{cm}^{2}$ and $6 \times 10^{17}$ ions $/ \mathrm{cm}^{2}$. Throughout this text the lower and higher dose implantations are referred to as case I and case II, respectively. Details of the implantation conditions are summarized in Table I.

The implanted silicon samples were analyzed using SAM to assess the dose and depth of the implanted ions at various positions on the surface of the wedge. The measured

TABLE I. Details of the implantation parameters used for the two implantation conditions used in the present study.

\begin{tabular}{ccc}
\hline \hline Case/Parameter & Case I & Case II \\
\hline Pulse width & $10 \mu \mathrm{s}$ & $21 \mu \mathrm{s}$ \\
Rise time & $6 \mu \mathrm{s}$ & $8.5 \mu \mathrm{s}$ \\
Dose pcr pulse & $2 \times 10^{10} \# / \mathrm{cm}^{2}$ & $2.1 \times 10^{11} \# / \mathrm{cm}^{2}$ \\
Number of pulses & $8.46 \times 10^{6}$ & $3.45 \times 10^{6}$ \\
Current density & $1.52 \mu \mathrm{A} / \mathrm{cm}^{2}$ & $7.0 \mu \mathrm{A} / \mathrm{cm}^{2}$ \\
Sec. elec. coeff. $(\gamma)$ & 6 & 6 \\
Target bias & $-50 \mathrm{kV}$ & $-50 \mathrm{kV}$ \\
Gas pressure & $2 \times 10^{-4} \mathrm{Torr}$ & $4 \times 10^{-4} \mathrm{Torr}$ \\
Applied dose & $2 \times 10^{17} \# / \mathrm{cm}^{2}$ & $8 \times 10^{17} \# / \mathrm{cm}^{2}$ \\
Retained dose & $1.5 \times 10^{17} \# / \mathrm{cm}^{2}$ & $6 \times 10^{17} \# / \mathrm{cm}^{2}$ \\
\hline \hline
\end{tabular}

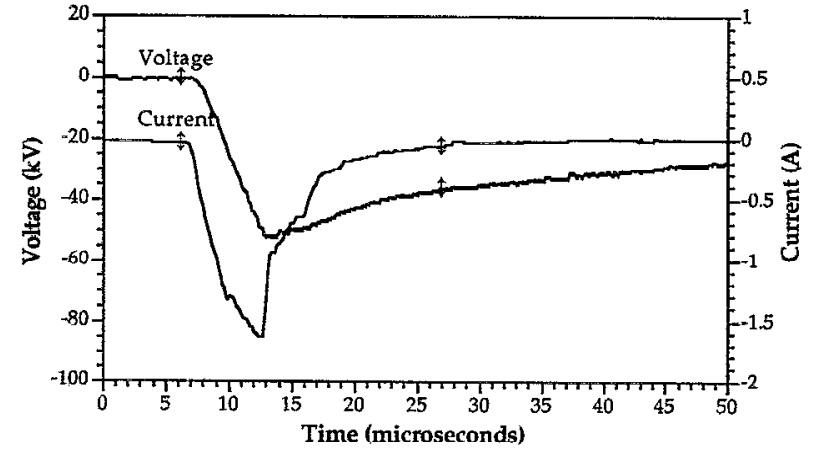

FIG. 2. Oscillographs showing the target bias pulse for the implantation conditions used in case $\mathrm{I}$.

depth profiles of the implanted nitrogen were compared with simulations from a Monte Carlo computer code, TAMIX (transport and mixing from ion irradiation). ${ }^{10} \mathrm{~A}$ Tencor Alpha-Step profilometer was used to measure the sputtered depth along the plane of the wedge.

\section{RESULTS AND DISCUSSION}

Figures 2 and 3 show oscillographs for the target bias applied in case I and case II, respectively. The arrows on the curves represent integration time durations. It is clear from Table I that the ratio of the rise time and the total pulse width are different for the two cases. This ratio dictates the fractional energy distribution of incident ions impinging the target. In order to effectively compare the measured and calculated depth profiles of the implanted ions, the fractional energy distribution of the incident ions stemming from the shape of applied bias pulse is necessary. A PSII code developed by Emmert et al. ${ }^{11}$ to simulate the energy distribution of incident ions was applied for the two implantation conditions. Results of this simulation as applied to the present study are shown in Fig. 4. For case I, the percentage of ions impacting the target with full energy is $30 \%$ and for case II it is $40 \%$, for the estimated ratios of rise time to total pulse width (from Table I) are $65 \%$ and $40 \%$ for case I and case II,

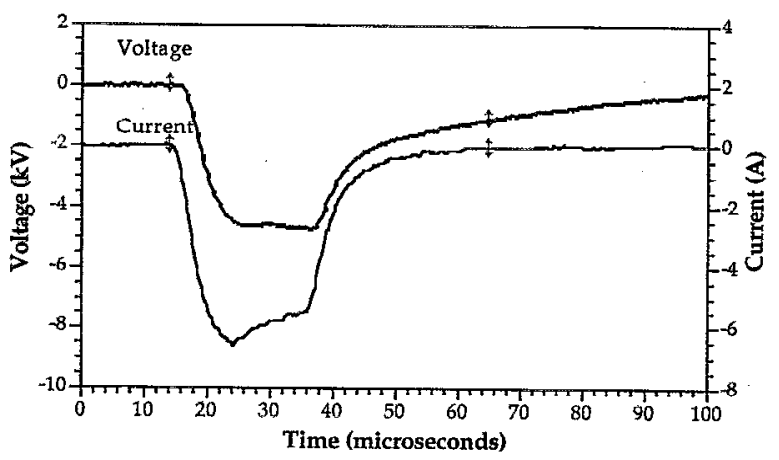

FIG. 3. Oscillographs showing the target bias pulse for the implantation conditions used in case II. 


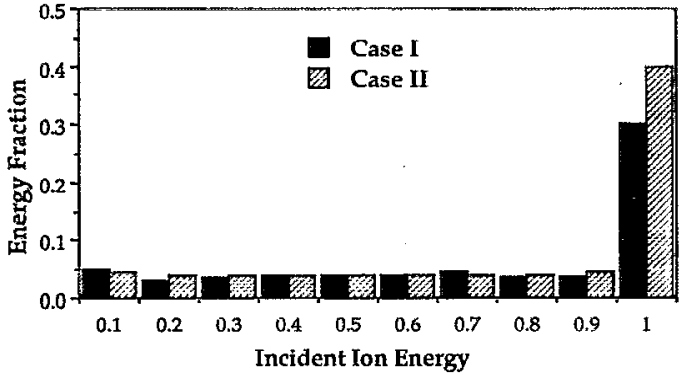

FIG. 4. Incident ion energy distribution fraction due to the rise time of the applied pulse as calculated by the PSII code of Emmert et al. (see Ref. 10).

respectively. It is also clear that the pulse with longer duration at full bias has more ions impinging with full energy.

In order to apply the TAMIX computer simulation to calculate the retained dose, the determination of the ion species ratios in the plasma is essential. This is because the ratio of ions impinging with full energy depends strongly on the relative concentration of $\mathrm{N}^{+}$and $\mathrm{N}_{2}^{+}$ions in the plasma. This is illustrated in Fig. 5 which shows the plasma code simulations ${ }^{11}$ of the incident ion's fractional energy distribution for plasmas with $100 \% \mathrm{~N}^{+}$and $25 \% \mathrm{~N}^{+}$, for the pulse shape applied in case I. For conditions listed in Table I, an ion composition of $75 \% \mathrm{~N}_{2}^{+}$and $25 \% \mathrm{~N}^{+}$has been used, as previously measured by Tang et $a l^{12}$ The incident ion energy distribution for $25 \% \mathrm{~N}^{+}$as determined in Fig. 5, was used as input for TAMIX simulations to predict the retained dose. Figures 6 and 7 show the comparison of the depth profiles for the TAMIX simulations with experimentally determined SAM results for the two implantation conditions. However, for case I the calculated depth profile is slightly greater than the measured depth profile while for case II the trend is reversed. The SAM results also show a peak concentration of nitrogen nearer to the surface for case I. This is presumably due to the effect of dose rate per pulse which is a factor of 10 higher for case II and may have resulted in some thermal diffusion in this case.

To determine the effect of target edges on the retained dose, systematic SAM analyses were performed on the silicon samples that were affixed along the face of the wedge. Figure 8(a) (case I) and Fig. 8(b) (case II) show the results of

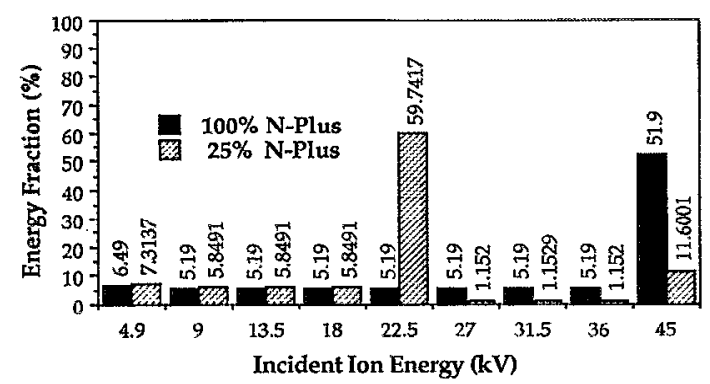

FIG. 5. Incident ion energy distribution fraction due to ion species ratio in plasma as determined by the PSII code of Emmert et al. (see Ref. 10).

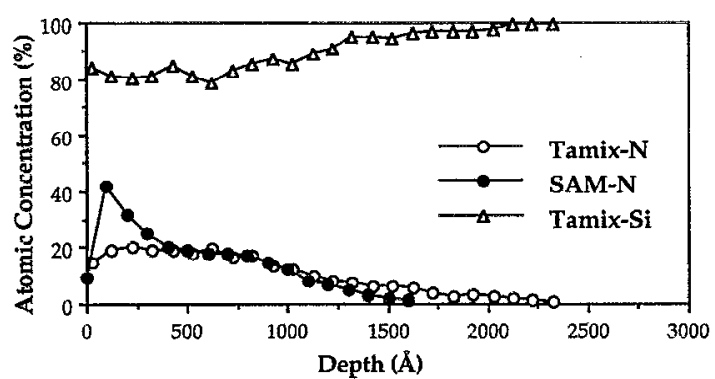

FIG. 6. TAMXX simulations and SAM depth profiles for nitrogen implanted into silicon, for case I

these measurements on the surface of the wedge conceptually unfolded around $30^{\circ}$ angle edge. Two points are worthy of attention: (1) the edges in the neighborhood of the $30^{\circ}$ angle have a lesser retained dose as compared to the $90^{\circ}$ angle edge and (2) the faces of the wedge with smaller area have, on the average, higher dose.

The observation of smaller retained doses at the edges shown in Figs. 8(a) and 8(b) are consistent with the simulations performed by Watterson, ${ }^{7}$ where it was observed that the incident ion fluence for square and knife edge target lead to ion trajectories similar to those shown in Fig. 9. It is obvious from this figure that the ratio of the incident ions with grazing angles to those impinging at normal angles is higher at the sharper edges. Consequently, a lower retained dose and enhanced sputtering are expected at edges contained by acute angles. A two-dimensional fluid simulation performed by Hong and Emmert predicts similar trends. ${ }^{13}$

The observation of higher dose accumulation on smaller area is analogous to the experimental observation of Collins and Tendys, ${ }^{14}$ where it was noted that for larger diameter spheres the ion drain on the plasma outside the sheath is more severe. The explanation forwarded for this observation is that the sheath will keep expanding to replenish ions for the sphere with larger diameter but the sheath for sphere with smaller diameter will be stationary at earlier time scale of sheath propagation. In a similar manner, different surface areas of the wedge may push the expanding sheath to different positions.

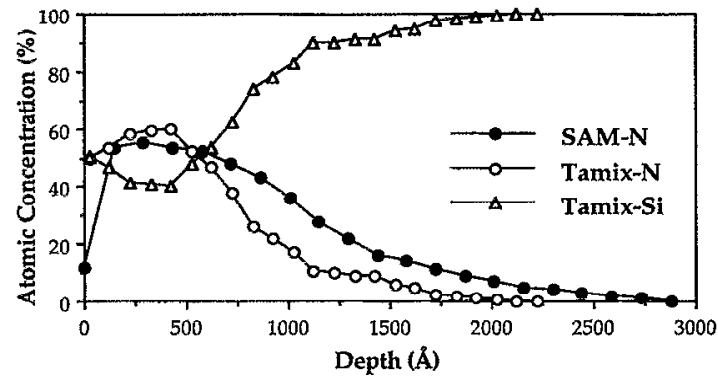

FIG. 7. TAMTX simulations and SAM depth profiles for nitrogen implanted into silicon, for case II. 

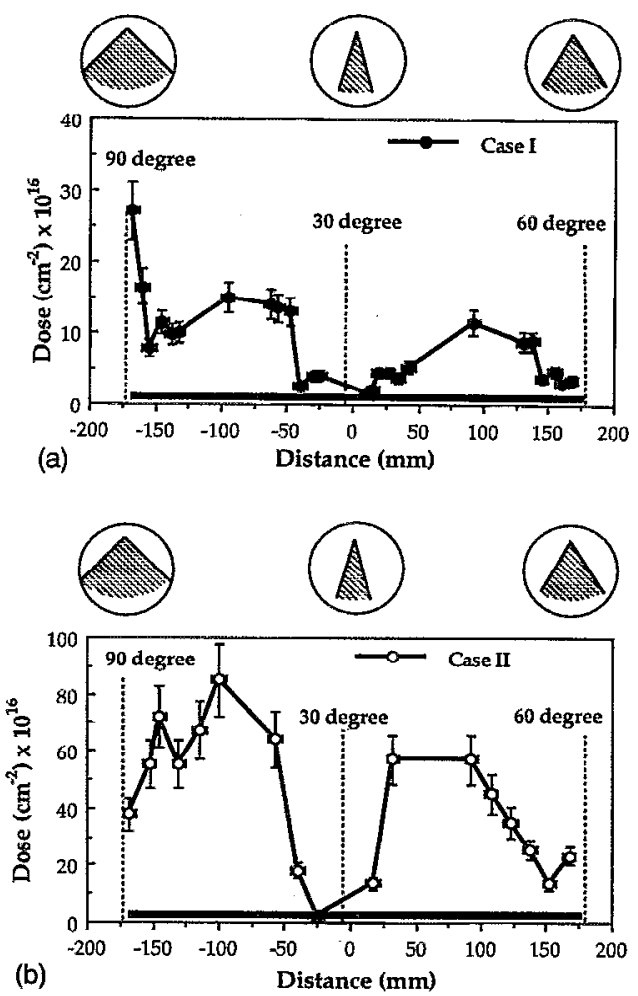

FIG. 8. Measured dose on the wedge surface for (a) case I and (b) case II. The vertical dotted lines show edge position and the dark horizontal solid line represents the linear dimension on the wedge surface when conceptually unfolded at the $30^{\circ}$ angle.

To better understand the findings of Fig. 8, silicon samples corresponding to case I were investigated further. Figure 10(a) represents the retained dose for case I on the surface of the wedge. It was noted that there is higher retained dose in the vicinity of the normal angle as compared to acute angle. The lesser dose retention near the acute angle is attributed to enhanced sputtering and shallow depths of the implanted ions. Figure 10(b) shows a magnified representa-

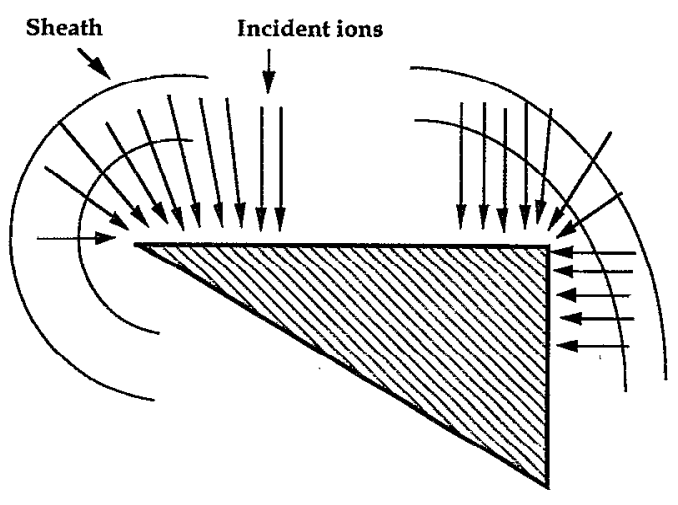

FIG. 9. A conceptual configuration of incident ion fluence at the edges of the wedge as determined by the simulations of Watterson (see Ref. 7).
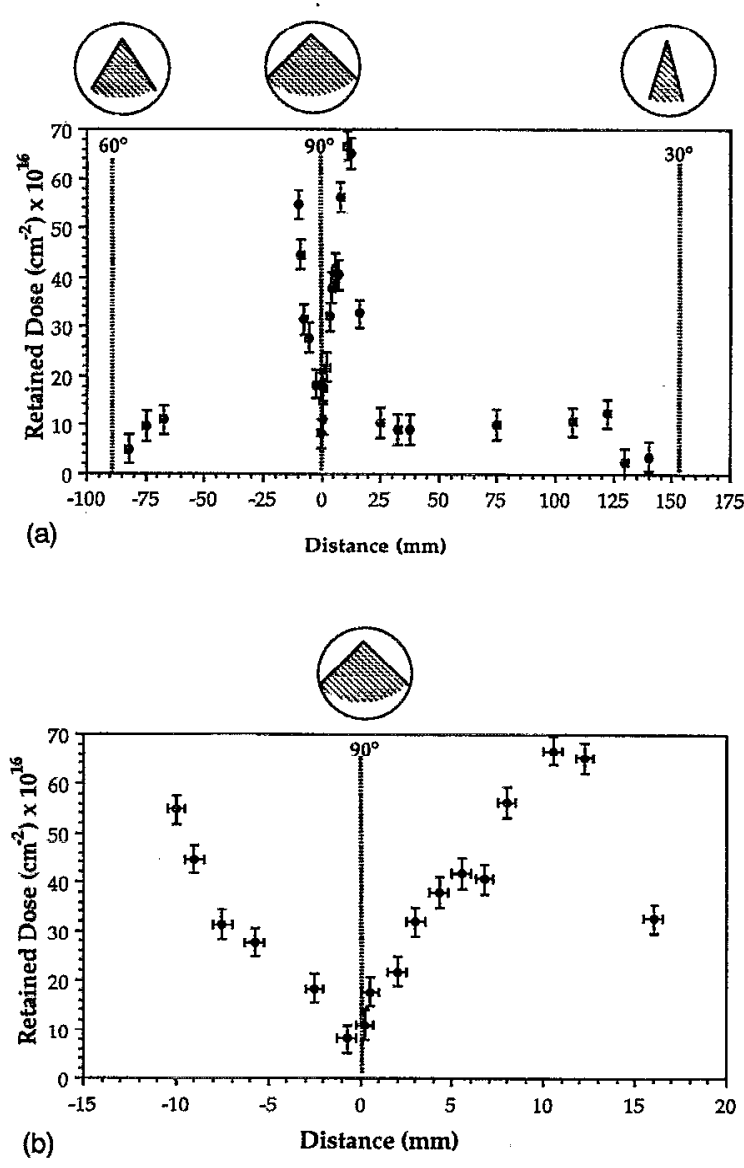

FIG. 10. (a) Measured dose on the wedge surface for the case II, where the wedge has been unfolded at the $90^{\circ}$ angle. (b) A close-up of (a) showing detailed information at the $90^{\circ}$ edge.

tion in the vicinity of the $90^{\circ}$ angle edge. There is lesser dose right at the edge which can be attributed to the non-normal incident ions.

The maximum depth of penetration of the implanted ions into the target depends on the energy of the incident ions and the angle of incidence with target plane. In our study, the

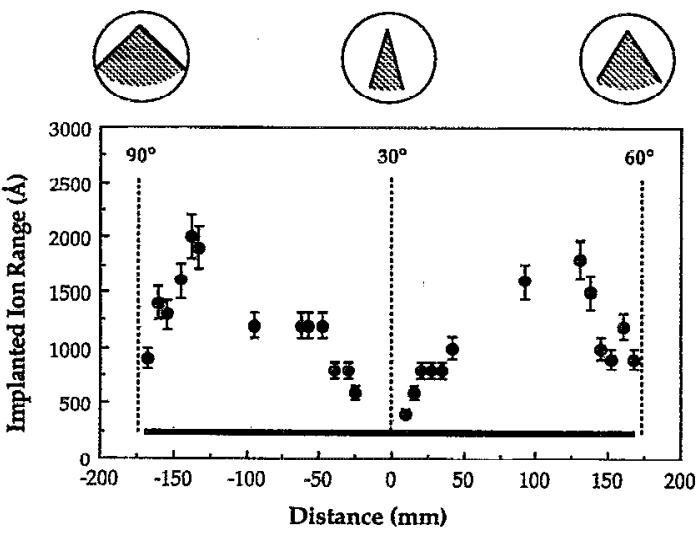

FIG. 11. Measured ion ranges into silicon at various positions on the wedge surface. 


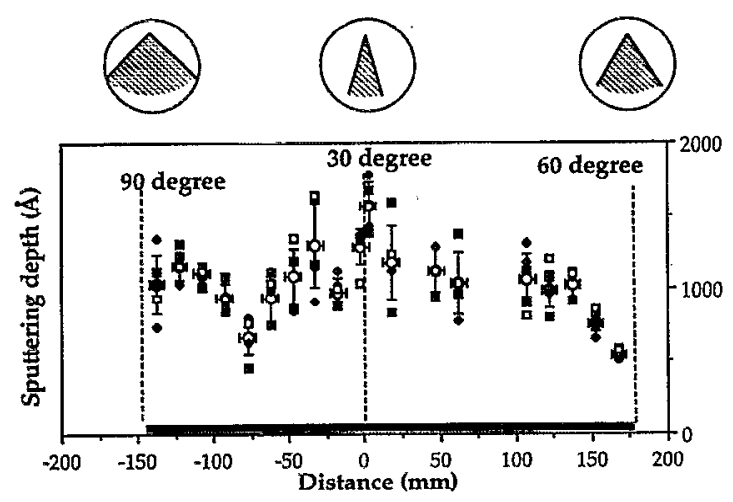

FIG. 12. The sputtered depth at various locations on the surface of the wedge.

ions strike at grazing angle in the vicinity of acute angle edges so the final range of the implanted ions is reduced with a factor of cosine of the angle of the ion incidence. Figure 11 shows the final implanted depth of the nitrogen ions into the silicon samples as determined by SAM measurements. It is obvious that the ions penetrate deeper near the normal angle edge which compliment the previously described understanding. Figure 12 shows the profile of sputtered depth during ion implantation. As predicted by theory a relatively higher sputtering trend is noted near the sharp angles.

\section{CONCLUSIONS}

PSII of the wedge-shaped target has revealed that there was enhanced sputtering, less retained dose, and a shallower penetration range of implanted ions in the vicinity of the acute angle edges as compared to the square angle edge, a finding that is in agreement with previous investigations (Refs. 6 and 7). It was noted that the wedge face with smaller area retained, on the average, a higher dose as compared to the face with larger area. This difference in dose may be due to the stopping of the sheath at different positions as well as the difference in ionization rates of neutrals inside the sheath due to different electric field intensities. The sheath edge propagates to a greater extent on the side corresponding to the wedge face with larger area compared to the side corresponding to the smaller area. The higher ionization rate along the face with smaller area may be due to convergence of electric field lines which enhance the plasma density and consequently, the sheath stops at an earlier time scale. Successful implantation of knife edges can be achieved by judicious arrangement of the targets or by extension of target geometry so that a matching continuous planar surface can be attained.

\section{ACKNOWLEDGMENTS}

The authors are grateful to Professor N. Hershkowitz and Professor G. A. Emmert for useful discussions during the course of this work. The assistance provided by Patricia Doyle, James Firmiss, and Nathaniel Horswill is sincerely appreciated. The work was supported by NSF Grant No. ECD 8721545, DOE CRADA Agreement No. 9-XX3-8362E-1, and U.S. Army Grant No. DAAL 03-90-G-0124.

'J. R. Conrad, U. S. Patent No. 4764 394, August (1988).

${ }^{2}$ J. R. Conrad, R. A. Dodd, S. Han, M. Madapura, J. Scheuer, K. Sridharan, and F. J. Worzala, J. Vac. Sci. Technol. A 8, 3146 (1990).

${ }^{3}$ Proceedings of the "First International Workshop on Plasma-Based Ion Implantation," Madison, WI Aug. 4-6, 1993; J. Vac. Sci. Technol. B 12, 807 (1994).

${ }^{4}$ J. T. Scheuer, M. Shamim, and J. R. Conrad, J. Appl. Phys. 67, 1241 (1990).

${ }^{5}$ M. Shamim, J. T. Scheuer, and J. R. Conrad, J. Appl. Phys. 69, 2904 (1991).

${ }^{6}$ Shamim M. Malik, R. P. Fetherston, K. Sridhran, and J. R. Conrad, Plasma Sources Sci. Technol. 2, 81 (1993).

${ }^{7}$ P. A. Watterson, J. Phys. D 22, 1300 (1989).

${ }^{8}$ Shamim M. Malik, K. Sridhran, R. P. Fetherston, A. Chen, and J. R. Conrad, J. Vac. Sci. Technol. B 12, 843 (1994).

${ }^{9}$ P. D. Goode and I. J. R. Baumvol, Nucl. Instrum. Methods 189, 161 (1981).

${ }^{10}$ S. N. Han, G. L. Kulcinski, and J. R. Conrad, Nucl. Instrum. Methods B 45, 701 (1990).

${ }^{11}$ G. A. Emmert and M. A. Henry, J. Appl. Phys. 71, 113 (1992).

${ }^{12}$ B. Y. Tang, R. P. Fetherston, M. Shamim, R. A. Breun, A. Chen, and J. R. Conrad, J. Appl. Phys. 73, 4176 (1993).

${ }^{13}$ MunPyo Hong and G. E. Emmert, J. Vac. Sci. Technol. B 12, 889 (1994).

${ }^{14}$ G. A. Collins and J. Tendy, Plasma Sources Sci. Technol. 3, 10 (1994). 